\title{
Answer to the Letter to the Editor of LJ Castro-Alves et al. concerning "Comparative study of the efficacy of transdermal buprenorphine patches and prolonged-release tramadol tablets for postoperative pain control after spinal fusion surgery: a prospective, randomized controlled non-inferiority trial" by HJ Kim et al. (2017) Eur Spine J;26:2961-2968
}

\author{
Ho-Joong Kim ${ }^{1}$
}

Received: 25 September 2018 / Accepted: 1 October 2018 / Published online: 10 October 2018

(c) Springer-Verlag GmbH Germany, part of Springer Nature 2018

Dear Editor,

We are grateful for these excellent comments. Our answers are below:

Firstly, it is not clear if intraoperative analgesic administration was standardized using a specific criterion. This has been shown to alter the primary outcome [6].

Thank you for your excellent comments. We agree with this opinion. In this clinical trial, however, we did not use any intraoperative analgesics.

Secondly, the authors do not mention if the use of any postoperative multimodal analgesic was standardized between the groups as this could also have affected the primary outcome.

We appreciate this comment. For postoperative pain control, patient-controlled analgesia (PCA) and buprenorphine/tramadol were used and any other different analgesics were not used.
Lastly, it is likely that patients titrated analgesics to a low pain score and this may explain the lack of difference among pain scores between the study groups.

Thank you for this excellent comment. Below is the protocol in the tramadol group.

"In the Tramadol group, a sustained action oral tramadol dose of $150 \mathrm{mg} /$ day was provided $36 \mathrm{~h}$ postoperatively. The possible doses of tramadol tablets were 150, 200, 250 , and $300 \mathrm{mg} / \mathrm{day}$. Supplementary acetaminophen was available throughout the study as rescue medication."

As you mentioned, titration was allowed for patient's safety. However, the strength of $120 \mathrm{mg} /$ day of tramadol is the equivalent to that of 7 -day patch of $5 \mathrm{mcg} / \mathrm{h}$. Therefore, titration would not influence the present results.

\section{Compliance with ethical standards}

Conflict of interest The author declare that there is no conflict of interest.
Ho-Joong Kim

oshjkim@gmail.com; oshjkim@snu.ac.kr

1 Spine Center and Department of Orthopedic Surgery, Seoul National University College of Medicine, Seoul National University Bundang Hospital, 166 Gumi-ro, Bundang-gu, Sungnam-si, Gyeonggi-do 463-707, Republic of Korea 